\title{
QoS Routing using Energy Parameter in Mobile Ad Hoc Network
}

\author{
Rajgopal .G \\ SITE, VIT University \\ Vellore, India
}

\author{
Manikandan .K \\ SCSE, VIT University \\ Vellore, India
}

\author{
Sivakumar.N \\ SITE, VIT University \\ Vellore, India
}

\begin{abstract}
A Mobile Ad Hoc Networks (MANET) is a collection of mobile nodes that can communicate with each other using wireless links without utilizing any fixed based station infrastructure and centralized management. In the present advanced technology there are many developments in Mobile Ad-hoc Networks (MANET) protocols. MANET is the wireless technology used in various applications like military, mobile devices etc, so improving the performance is an advantage in it. There are different parameters taken from MANET like routing, energy consumption, latency, bandwidth, traffic, packet loss etc. In this way there are many parameters taken as issue and new protocols are designed. This paper discusses the performance and comparison of different routing protocols of Mobile ad hoc networks based on the energy level. To reduce the energy consumption in AODV, DSR, we proposed enhanced AODV and enhanced DSR. Simulations are done using NS-2 and the results show that enhanced AODV and enhanced DSR consumes less energy compared to existing protocols and also comparison of energy consumption in each protocol.
\end{abstract}

\section{Keywords}

MANET, AODV, DSR, DSDV, OLSR.

\section{INTRODUCTION}

A mobile ad hoc network $[1,2]$ is an autonomous system consisting solely of mobile terminals connected with wireless links. This type of network has received considerable interest in recent years due to its capability to be deployed quickly without any fixed infrastructure. As mobile ad hoc networks provide the users unparalleled flexibility, they pose serious challenges to the designers. Due to the lack of a fixed infrastructure, nodes must self-organize and reconfigure as they move, join or leave the network. All nodes are essentially the same and there is no natural hierarchy or central controller in the network. All functions have to be distributed among the nodes. Nodes are often powered by batteries and have limited communication and computation capabilities. MANETs are useful in many applications because they do not need any infrastructure support. Collaborative computing and communications in smaller areas (building organizations, conferences, etc.) can be set up using MANETS. Communications in battlefields and disaster recovery areas are further examples of application environments. In the next section we discussed about routing protocols then compared routing protocols based on energy. Simulations are done using NS-2 and the results show that enhanced AODV and enhanced DSR consumes less energy compared to existing protocols.
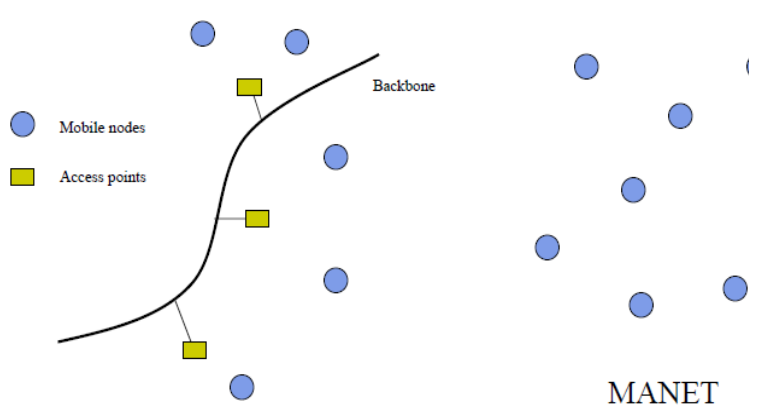

Wireless Mobile Network

Fig 1. Wireless network

\section{RELATED WORKS}

Routing in wireless ad hoc networks is clearly different from routing found in traditional infrastructure networks. Routing in ad hoc networks needs to take into account many factors including topology, selection of routing path and routing overhead, and it must find a path quickly and efficiently. Ad hoc networks generally have lower available resources compared with infrastructure networks and hence there is a need for optimal routing. Also, the highly dynamic nature of these networks means that routing protocols have to be specifically designed for them. In wireless ad hoc networks, the communication range of a node is often limited and not all nodes can directly communicate with one another. Nodes are required to relay packets on behalf of other nodes to allow communication across the network. Since there is no predetermined topology or configuration of fixed routes, an ad hoc routing protocol is used to dynamically discover and maintain up-to-date routes between communicating nodes. Routing protocols are divided into three types. They are proactive, reactive and hybrid routing protocols.

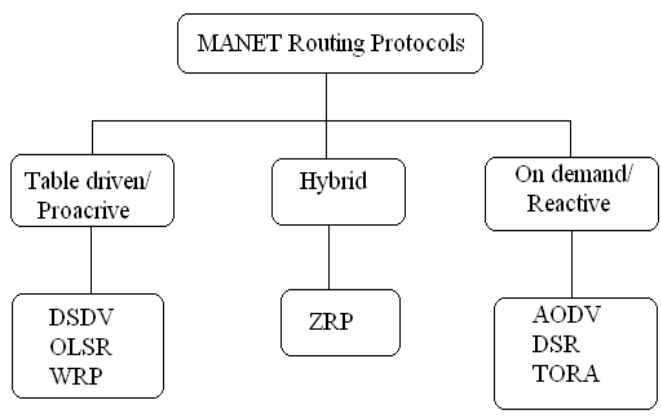

Fig 2. Routing Protocols 


\subsection{Proactive Routing Protocols}

Ad hoc routing protocols may generally be categorized into Proactive and reactive according to their routing strategy [3]. Proactive protocols require that nodes in a wireless ad hoc network should keep track of routes to all possible destinations so that when a packet needs to be forwarded, the route is already known and can be used immediately. Any changes in topology are propagated through the network, so that all nodes know of those changes in topology. Examples include "destinationsequenced distance-vector" (DSDV) routing [4], On-demand protocols only attempt to build routes when desired by the source node so that the network topology is detected as needed (on-demand). When a node wants to send packets to some destination but has no routes to the destination, it initiates a route discovery process within the network. Once a route is established, it is maintained by a route maintenance procedure until the destination becomes inaccessible or until the route is no longer needed. Examples include "ad hoc on-demand distance vector routing" (AODV) [5], "dynamic source routing" (DSR) [6]. Proactive protocols have the advantage that new communications with arbitrary destinations experience minimal delay, but suffer the disadvantage of the additional control overhead to update routing information at all nodes. To cope with this shortcoming, reactive protocols adopt the inverse approach by finding a route to a destination only when needed. Reactive protocols often consume much less bandwidth than proactive protocols [7], but they will typically experience a long delay for discovering a route to a destination prior to the actual communication. However, because reactive routing protocols need to broadcast route requests, they may also generate excessive traffic if route discovery is required frequently.

\subsubsection{DSDV Protocol:}

The Destination-Sequenced Distance-Vector (DSDV) Routing Algorithm [4] is a proactive hop-by-hop distance vector routing protocol, which is based on the idea of the classical BellmanFord Routing Algorithm with certain improvements. Every mobile station maintains a routing table that lists all available destinations, the number of hops to reach the destination and the sequence number assigned by the destination node. The sequence number is used to distinguish stale routes from new ones to avoid the formation of loops. The stations periodically transmit their routing tables to their immediate neighbors. A station also transmits its routing table if a significant change has occurred in its table from the last update sent. The update is both time-driven and event-driven. The routing table updates can be sent in two ways:

a "full dump" where the full routing table is sent to the neighbors (which could span many packets); or

an incremental update where only those entries from the routing table that have had a metric change since the last update are sent (and these must fit in a single packet).

If there is space in the incremental update packet, then those entries whose sequence number has changed may be included. When the network is relatively stable, incremental updates are sent to avoid extra traffic and full dumps are relatively infrequent. In a fast-changing network, incremental packets can grow large so full dumps will be more frequent. Each route update packet, in addition to the routing table information, also contains a unique sequence number assigned by the transmitter. The route labeled with the highest (i.e. most recent) sequence number is used. If two routes have the same sequence number then the route with the best metric (i.e. shortest route) is used. Based on past history, the stations estimate the settling time of routes. The stations delay the transmission of a routing update by settling time so as to eliminate those updates that would occur if a better route were found very soon.

\subsubsection{OLSR Protocol:}

Optimized Link State Routing (OLSR) is a routing protocol used for Mobile Ad-Hoc Networks (MANET). It is a best-effort proactive protocol. Proactive protocols are characterized by all nodes maintaining routes to all destinations at all times through the periodic exchange of protocol messages. This gives them the advantage of having pre-computed routes available when needed and to propagate topology changes in bulk updates to many nodes. OLSR performs hop-by-hop routing, where each node uses its most recent topology information for routing. OLSR is highly focused on reducing the protocol overhead. As a result, information about QoS-related state is not propagated throughout the network. But with the rising popularity of multimedia applications and the potential commercial usage of MANETs, QoS support in ad-hoc networks has become a very critical issue and a range of QoS signalling and routing protocols have been proposed.

OLSR [8] is an optimization over the classical link state protocol; OLSR minimizes the overhead from flooding of control traffic by using only selected nodes, called Multi Point Relays (MPRs), to retransmit control messages. This technique significantly reduces the number of retransmissions required to flood a message to all nodes in the network. Secondly, OLSR requires only partial link state to be flooded in order to provide shortest path routes. The minimal set of link state information required is that all nodes, selected as MPRs, must declare the links to their MPR selectors.

OLSR is designed to work in a completely distributed manner and does not depend on any central entity. The protocol does not require reliable transmission of control messages: each node sends control messages periodically, and can therefore sustain a reasonable loss of some such messages. Also, OLSR does not require sequenced delivery of messages. Each control message contains a sequence number which is incremented for each message. Thus the recipient of a control message can, if required, easily identify which information is more recent - even if messages have been re-ordered while in transmission.

\subsection{Reactive Routing Protocols}

Reactive protocols take a lazy approach to routing. In contrast to proactive routing protocols, all up-to-date routes are not maintained at every node, but instead the routes are created as and when required. When a source wants to send to a destination, it invokes the route discovery mechanisms to find the path to the destination. In this section several typical reactive (on-demand) routing protocols [9] are introduced.

\subsubsection{AODV Protocol:}

Ad hoc on-demand distance vector (AODV) routing [5] adopts both a modified on-demand broadcast route discovery approach used in DSR [6] and the concept of destination sequence number adopted from destination-sequenced distance-vector routing (DSDV)[4]. When a source node wants to send a packet to some destination and does not have a valid route to that destination, it initiates a path discovery process and broadcasts a route request 
(RREQ) message to its neighbors. The neighbors in turn forward the request to their neighbors until the RREQ message reaches the destination or an intermediate node that has an up-to-date route to the destination. Figure 3 illustrates the propagation of the broadcast RREQs in an ad hoc network.

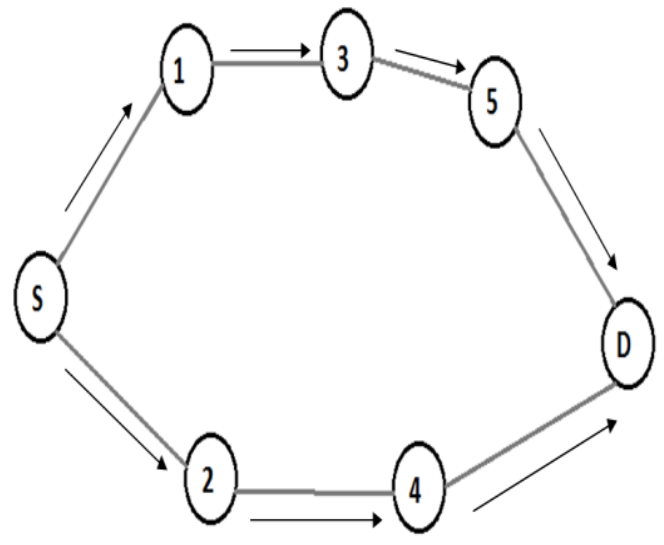

Fig 3. RREQ Propagation in AODV

In AODV, each node maintains its own sequence number and a broadcast ID. Each RREQ message contains the sequence numbers of the source and destination nodes and is uniquely identified by the source node's address and a broadcast ID. AODV utilizes destination sequence numbers to ensure loopfree routing and use of up-to-date route information. Intermediate nodes can reply to the RREQ message only if they have a route to the destination whose destination sequence number is greater or equal to that contained in the RREQ message. So that a reverse path can be set up, each intermediate node records the address of the neighbor from which it received the first copy of the RREQ message, and additional copies of the same RREQ message are discarded. Once the RREQ message reaches the destination (or an intermediate node with a fresh route) the destination (or the intermediate node) responds by sending a route reply (RREP) packet back to the neighbor from which it first received the RREQ message. As the RREP message is routed back along the reverse path, nodes along this path set up forward path entries in their routing tables (Figure 4).

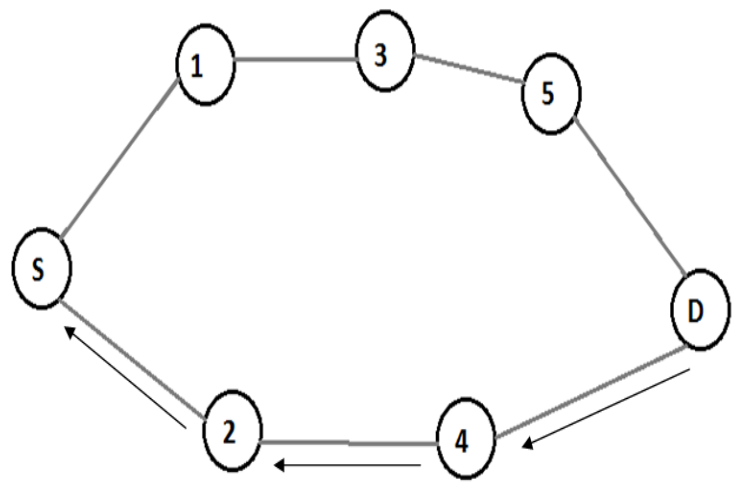

Fig 4. RREP Propagation in AODV
When a node detects a link failure or a change in neighborhood, a route maintenance procedure is invoked: If a source node moves, it can restart the route discovery procedure to find a new route to the destination. If a node along the route moves so that it is no longer contactable, its upstream neighbor sends a link failure notification message to each of its active upstream neighbors. These nodes in turn forward the link failure notification to their upstream neighbors until the link failure notification reaches the source node.

\subsubsection{DSR Protocol:}

Dynamic source routing (DSR) [6] is an on-demand routing protocol for wireless ad hoc networks. DSR is based on the concept of source routing, in which a source node indicates the sequence of intermediate routes in the header of a data packet. Like other on-demand routing protocols, the operation of DSR can be divided into two procedures: route discovery and route maintenance. Each node in the network keeps a cache of the source routes that it has learned. When a node needs to send a packet to some destination, it first checks its route cache to determine whether it already has an up-to-date route to the destination. If no route is found, the node initiates the route discovery procedure by broadcasting a route request message to neighboring nodes. This route request message contains the address of the source and destination nodes, a unique identification number generated by the source node, and a route record to keep track of the sequence of hops taken by the route request message as it is propagated through the network. When an intermediate node receives a route discovery request, it checks whether its own address is already listed in the route record of the route request message. If not, it appends its address to the route record and forwards the route request to its neighbors. Figure 5 illustrates the formation of the route record as the route request propagates through the network.

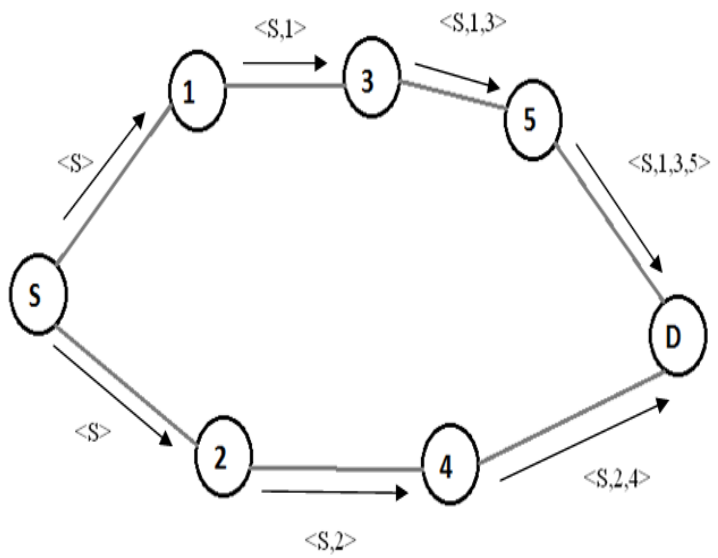

Fig 5. RREQ Propagation in DSR.

When the destination node receives the route request, it appends its address to the route record and returns it to the source node within a new route reply message. If the destination already has a route to the source, it can use that route to send the reply; otherwise, it can use the route in the route request message to send the reply. The first case is for situations where a network might be using unidirectional links and so it might not be possible to send the reply using the same route taken by the route request message. If symmetric links are not supported, the 
destination node may initiate its own route discovery message to the source node and piggyback the route reply on the new route request message. Figure 6 shows the transmission of route record back to the source node. Route maintenance uses route error messages and acknowledgement messages.

If a node detects a link failure when forwarding data packets, it creates a route error message and sends it to the source of the data packets. The route error message contains the address of the node that generates the error and the next hop that is unreachable. When the source node receives the route error message, it removes all routes from its route cache that have the address of the node in error. It may initiate a route discovery for a new route if needed. In addition to route error message, acknowledgements are used to verify the correct operation of links. To reduce the route search overhead, an important optimization is allowing an intermediate node to send a route reply to the source node if it already has an up to date route to the destination.

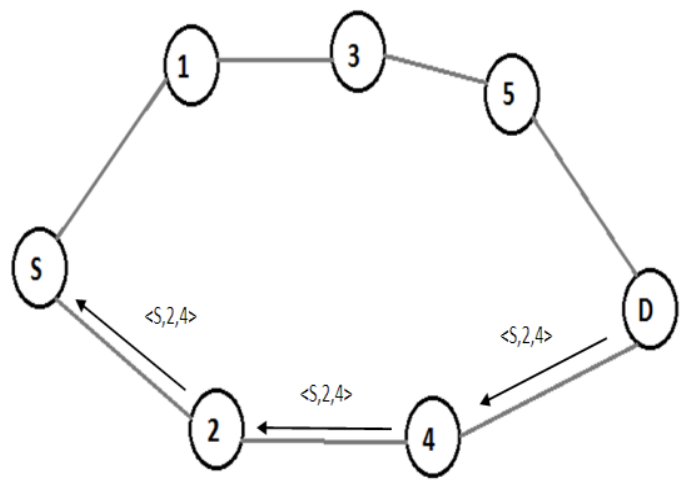

Fig 6. RREP Propagation in DSR.

\section{PERFORMANCE EVAlUATION OF PROTOCOLS BASED ON ENERGY}

Table 1. Simulation parameters

\begin{tabular}{|l|c|}
\hline PARAMETERS & VALUES \\
\hline Simulation time & $500 \mathrm{sec}$ \\
\hline Number of nodes & $20,50,75,100$ \\
\hline Traffic type & CBR \\
\hline Mobility model & $\begin{array}{c}\text { Random waypoint } \\
\text { model }\end{array}$ \\
\hline Packet size & 512 bytes \\
\hline MAC & Mac/802_11 \\
\hline Initial energy & $40 J$ oules \\
\hline Rxpower & 0.3 \\
\hline Txpower & 0.6 \\
\hline Protocols & $\begin{array}{l}\text { AODV, DSR, DSDV, } \\
\text { OLSR }\end{array}$ \\
\hline
\end{tabular}

Network simulators try to model the real world networks. The principal idea is that if a system can be modeled, then features of the model can be changed and the corresponding results can be analyzed. As the process of model modification is relatively cheap than the complete real implementation, a wide variety of scenarios can be analyzed at low cost. The simulations are done using Network Simulator [10].

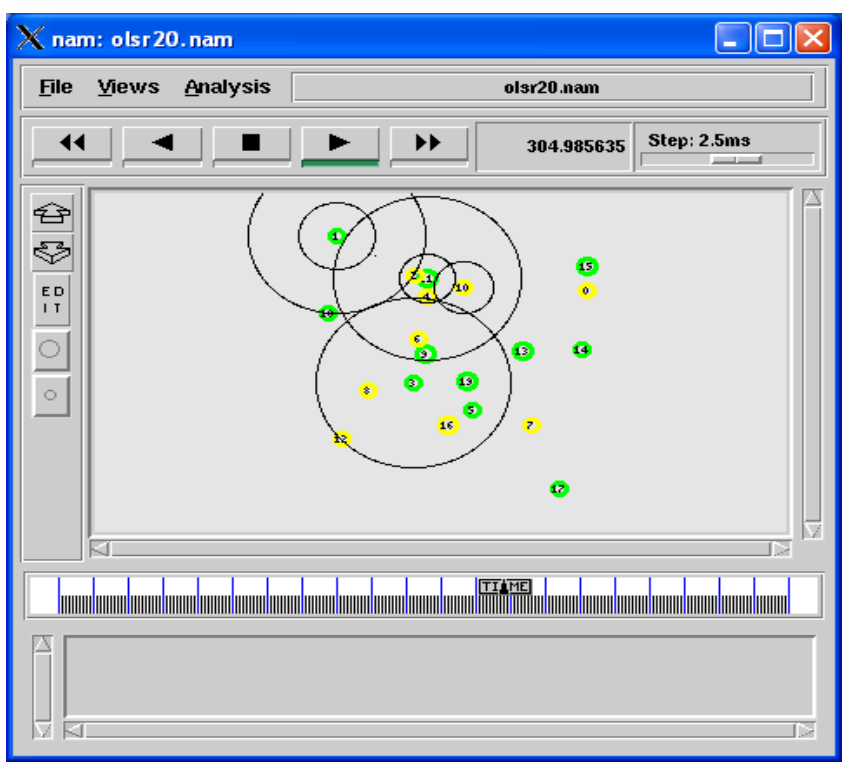

Fig 7. Simulation with 20 nodes after adding energy model

In this work, comparison of different routing protocols based on the energy is studied with simulations. Networks of 20,50,75 and 100 nodes are generated, where nodes roam in an area of 1000 by 1000 . Network traffic is generated by CBR source, where the source and the destination of a session are chosen randomly. The duration of the simulation is 500 seconds. In the figure 7 , we performed simulation using 20 nodes after adding energy model to the network. If the node contains more energy, the node will be in green color. If the node contains medium energy, it will be changed to yellow color. If the node contains no energy, it will be changed to red color.

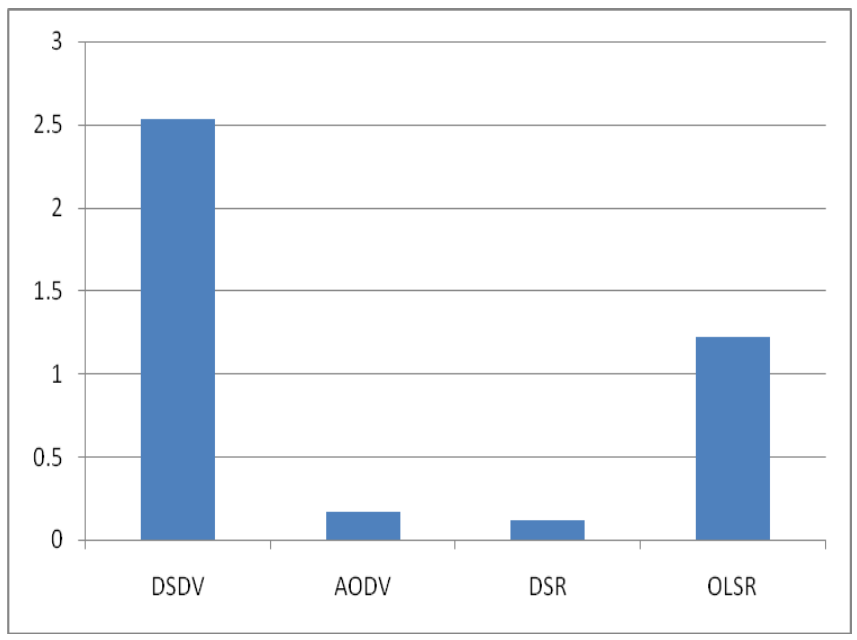

Fig 8. Remaining Energy (\%) after simulation 
Figure 8 shows energy consumption in each protocol when the number of nodes is 20 . Here, we calculated total remaining energy of network for each protocol after performing simulation. D.Kim et al [11] proposed formula to calculate energy level of node. The mobile node also loses some of it energy due to overhearing of the neighboring nodes. Thus, a node is losing its power over a period of time even if no data is being sent through it. Viewing all these factors a metric called Drain Rate (DR) was proposed in [11], Drain Rate of a node is defined as the rate of dissipation of energy of a node. Every node calculates its total energy consumption every $\mathrm{T}$ sec and estimates the DR, Actual Drain Rate is calculates by exponentially averaging the values of DRold and DRnew as follows: $\mathrm{DRi}=\alpha$ DRold $+(1-\alpha)$ DRnew. Where, $0<\alpha<1$, can be selected so as to give higher priority to updated information. Thus, higher the Drain Rate, faster the node is depleted of its energy. The total remaining energy is high in DSDV protocol compared to AODV, DSR, and OLSR. So, energy consumption is less in DSDV when the number of nodes is 20. DSR routing protocols consumes more energy compared to other protocols because the remaining energy in DSR is less. AODV also consumes more energy when compared to DSDV, OLSR and energy consumption is less compared to DSR. In OLSR, total remaining energy after simulation is $1.22 \%$ compare to DSR and AODV remaining energy is high but compare to DSDV remaining energy is low. So, energy consumption is less compared to AODV and DSR.

Table 2. Remaining energy (\%) in each protocol with different no. of nodes.

\begin{tabular}{|l|l|l|l|l|}
\hline $\begin{array}{l}\text { No. of } \\
\text { Nodes/ } \\
\text { Protocols }\end{array}$ & 20 & 50 & 75 & 100 \\
\hline DSDV & 2.53 & 2.6 & 8.5 & 8.7 \\
\hline AODV & 0.17 & 1.9 & 14.8 & 21 \\
\hline DSR & 0.12 & 1.3 & 17.6 & 24.2 \\
\hline
\end{tabular}

Here, we created topology using three different routing protocols in ns 2 and generated trace files. From the trace files, extracted the information using the Perl language and calculated the energy of total nodes and from that calculated total remaining energy of network. From the above results, DSDV protocol consumes more energy and the remaining energy is $88.7 \%$ when the number of nodes is 100 . For 20 nodes DSR protocol consumes more energy and remaining energy is $0.12 \%$. For 50 nodes, DSDV protocol consumes less energy and the remaining energy is $2.6 \%$ but for 75 nodes DSDV protocol consumes more energy and the remaining energy is $8.5 \%$.

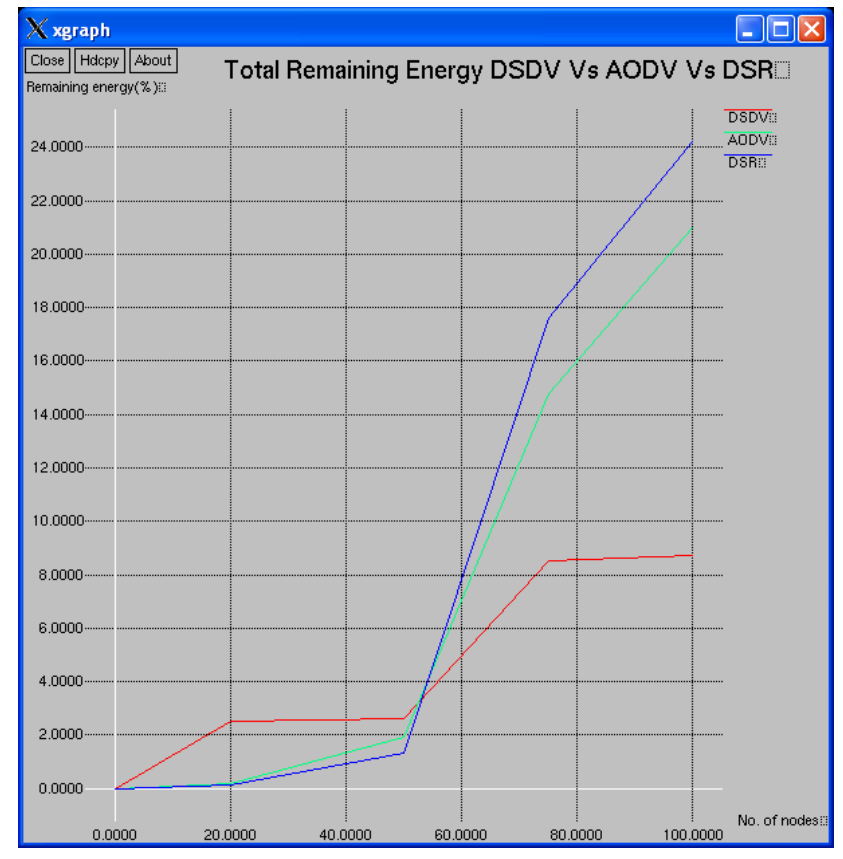

Fig 9. XGRAPH that shows Remaining energy in AODV, DSR, DSDV for different no. of nodes.

The figure 9 shows comparison of remaining energy in AODV, DSR, and DSDV when the number of nodes is 20, 50, 75 and 100 . When the number of nodes is 20 and 50, DSDV consumes less energy compare to DSR and AODV but, when the number of nodes is 75 and 100, energy consumption is high in DSDV compare to AODV and DSR. In DSDV, each node contains routing information about other nodes. If any node moves from one place to another place, the routing table of each node in the network will be updated with new path. DSDV consumes more energy when the number of nodes is 75 and 100 because mobility of nodes. Each node should update its routing table when a node moves. So the node will consume more energy. If number of nodes increases, mobility will also increase. Due to high mobility of nodes, DSDV consumes more energy compare to AODV and DSR.

\subsection{Proposed Method}

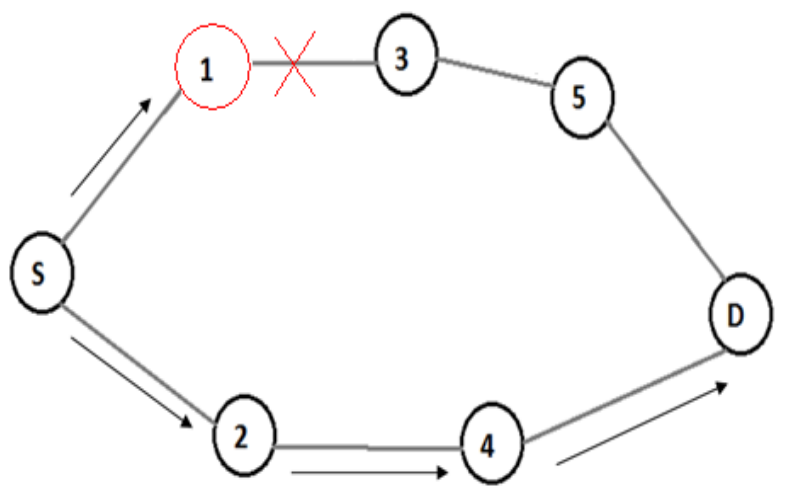

(a) RREQ Propagation in AODV. 


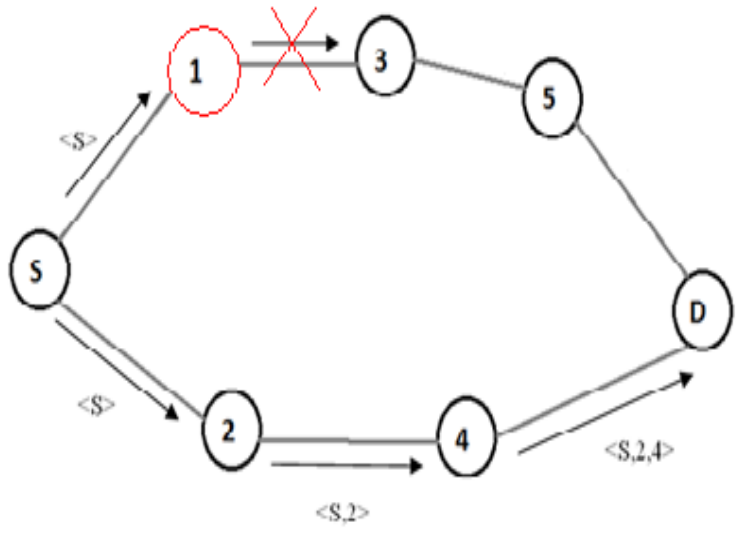

(b) RREQ Propagation in DSR.

Fig 10. Route Discovery Process.

From the above table 2 and figure 9 we can say that AODV and DSR routing protocols are consuming more energy when the number of nodes are 20 nodes. As a result of the comparative study [12] in various on demand routing protocols, we can say that each node get RREQ packet and each node will forward the RREQ packet as shown in figure 3 and figure 5. So, nodes which are having less energy and which are having more energy also get the RREQ packet. After some time the node which is having less energy will be going to shutdown. If we send the packet through the node which is going to be shutdown, the packet will be lost. In proposed method, the node which is having less energy (1\%) will not forward the RREQ packet. In the figure 10, node 1 is having less energy (1\%) will not forward the RREQ packet. In modified AODV and DSR, node 3 and 5 save the energy compare to figure 3 and figure 5 . By using this we can reduce the energy consumption in the network.

\subsection{Comparative study between DSR and AODV}

There are a few differences between DSR and AODV routing protocols [13]. Firstly, compared with DSR, the source node of AODV only knows the route to the destination, but in DSR, source node knows the route to intermediate node also. On the other hand, because in DSR, each data packet has to take the whole route information in the header, it costs large overhead which will waste data rate. [14]

Another difference between DSR and AODV is the usage of timer. In the DSR protocol, there is no timer used for the validation of routes. Stale routes could be used for routing. In AODV, timer is used for the freshness of a route. In DSR, with stale route, it is possible that the route is not validated. It will cause the loss the packets before source node is notified that the route is invalid. On the other hand, if the route is still valid, route overhead is saved for route discovery process.

In AODV, route which has not been used for a period of expire time will be deleted. The set of expire time is important since short expire time may lead to the deletion of still valid route and long expire time will give more non-fresh routes. Thirdly, it is about RREP. The destination of DSR could send RREQ more than once for the same RREQ without considering whether the same RREQ had been replied just a second ago for other routes.
In addition, multiple route entry could be store in the route table for the same destination in the same source node. That is, a source node has multiple route entries for one destination. While, in AODV, only one route reply can be sent by the destination and only one route entry per destination is stored in the route table.

The advantage of having more than one route entry per destination is that it can provide backup routes when there is a break on the current route. It means that there is no need to send a RREQ again to search for the route. On the other side, if the network has high mobility, the freshness of the backup route could be a problem and reinitiate a new RREQ should be a better choice. With the above analysis, it is found that AODV protocol should perform better in high mobility ad hoc network.

\section{RESULTS}

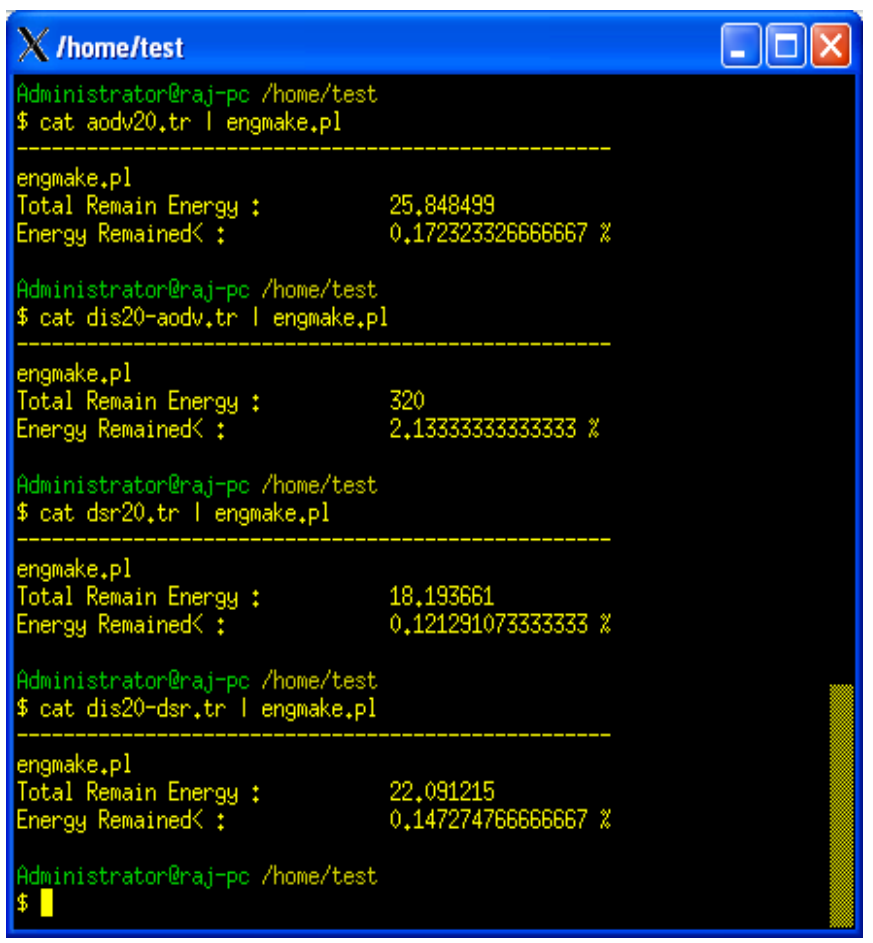

Fig 11. Total Remaining Energy calculation between basic and modified protocols.

In the figure 11, we are comparing total remaining energy of basic routing protocols with modified routing protocols. Total remaining energy in AODV is $0.17 \%$ when the numbers of nodes are 20. After implementing the proposed method remaining energy in modified AODV is $2.13 \%$. The energy consumption is reduced after implementing the proposed method. In DSR routing protocol, total remaining energy is $0.12 \%$ when the numbers of nodes are 20 . In modified DSR, remaining energy is $0.14 \%$. So, after implementing method energy consumption is decreased. The figure 12 shows the comparison of AODV, DSR with modified protocols based on energy. 


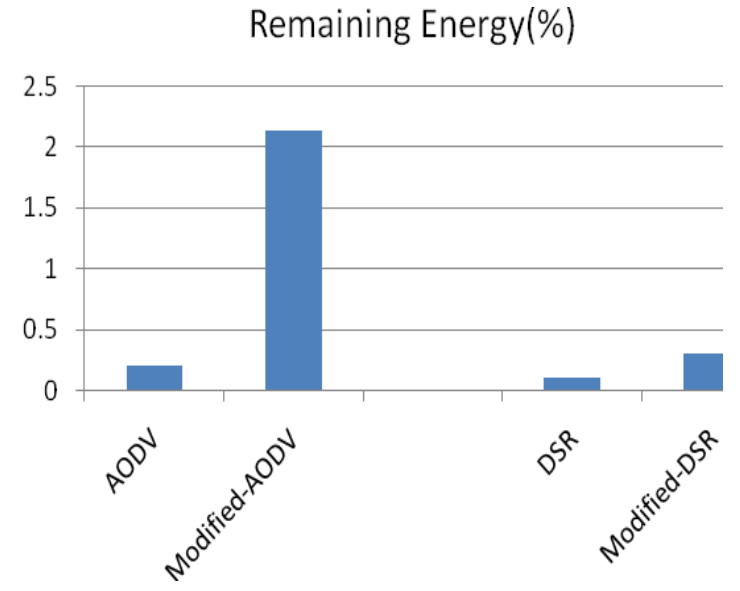

Figure 12. Remaining Energy comparison.

\section{CONCLUSION}

In this work, we have compared performance of routing protocols based on the energy level and proposed method is useful to save the energy in mobile ad hoc network. Here, nodes which are having less energy will not forward the RREQ packet. By using this method, we can decrease the energy consumption. In the future, we can reduce the energy consumption by using the same energy efficient path instead of finding the route again. The future scope of this paper is to implement the same concept in other protocols also.

\section{REFERENCES}

[1] D. D. Perkins, H. D. Hughes \& C. B. Owen, (2002) "Factors Affecting the Performance of Ad Hoc Networks," Proceedings of the IEEE International Conference on Communications (ICC), 2002, pp.2048-2052.

[2] Imrich Chlamtac, Marco Conti, Jennifer J.-N. Liu, (2003)" Mobile Ad hoc networking imperatives and challenges", Ad Hoc Networks, Vol 1, pp.13-64.

[3] Charles E. Perkins, Ad Hoc Networking, ed. C. E. Perkins, ISBN: 0-201-30976-9, Publisher: Addison Wesley Professional, 2001.

[4] C. E. Perkins and P. Bhagwat, Highly Dynamic Destination-Sequenced Distance-Vector Routing (DSDV) for Mobile Computers, In Proceedings of ACM SIGCOMM, pages 234-244, 1994.
[5] Charles E. Perkings, Elizabeth M. Belding-Royer, Samir R.Das, Ad Hoc On-Demand Distance Vector (AODV) Routing, http://www.ietf.org/internet-drafts/draft-ietfmanet-aodv-13.txt, IETF Internet draft, Feb 2003.

[6] J.Broch, D.Johnson, and D. Maltz, The Dynamic Source Protocol for Mobile Ad hoc Networks, http://www.ietf.org/internet-drafts/draft-ietf-manet-dsr10.txt, IETF Internet draft , 19 July 2004.

[7] Y.Ge,T.Kunz,L.Lamont: Proactive QoS routing in ad-hoc networks, in: Proceedings of the $2^{\text {nd }}$ International Conference on Ad-Hoc Networks and Wireless, Montreal, Canada, October2003.

[8] T.Clausen, P.Jacquet: Optimized link state routing protocol, Hypercom project, INRIA, France IETF RFC 3626,2003.

[9] A. Boukerche, "A simulation based study of on-demand routing protocols for ad hoc wireless networks," in Proceedings of 34th Annual Simulation Symposium, April 2001, pp. 85-92.

[10] The network simulator $\quad$ - ns-2. http:// www.isi.edu/nsnam/ns/.

[11] D.Kim, Garcia-Luna-Aceves, J.J. Obraczka, K. Cano \& J.C. Manzoni, P.(2003) "Routing mechanisms for Mobile Ad hoc Networks Based on Energy Drain Rate", Mobile Computing, Vo12, page(s): 161- 173.

[12] LiLayuan, LiChunlin, YaunPeiyan: Performance evaluation and simulations of routing protocols in ad hoc networks, doi:10.1016/j.comcom.2007.02.015, February 2007.

[13] Arun Kumar B. R. Lokanatha C. Reddy, Prakash S.Hiremath: Performance comparison of wireless routing protocols. International Journal of Computer Science and Network Security, VOL.8 No.6, June 2008.

[14] J. Broch, D.A. Maltz, D.B. Johnson, Y.C. Hu, and J.Jetcheva, A Performance Comparison of Multi-Hop Wireless Ad Hoc Network Routing Protocols, In Proceedings of the Fourth Annual ACM/IEEE International Conference on Mobile Computing and Networking (MobiCom'98), October 25-30, 1998, Dallas, Texas, USA, pp1- 13 .

[15] Ad hoc protocols implementations, http://www.wikipedia.org/wiki/ 